\title{
TRAVEL TIME FORECASTING AND DYNAMIC OD ESTIMATION IN FREEWAYS BASED ON BLUETOOTH TRAFFIC MONITORING
}

\author{
J.Barceló $^{(1)}$, L.Montero $^{(1)}$, L. Marqués ${ }^{(1)}$, P. Marinelli ${ }^{(2)}$ and C. Carmona ${ }^{(1)}$ \\ ${ }^{(1)}$ Department of Statistics and Operations Research and CENIT \\ (Center for Innovation in Transport) Technical University of Catalonia \\ (lidia.montero, laura.marques, jaume.barcelo, carlos.carmona)@upc.edu \\ ${ }^{(2)}$ Universitá di Roma, La Sapienza
}

\begin{abstract}
From the point of view of the information supplied by an ATIS to the motorists entering a freeway of one of the most relevant is the Forecasted Travel Time, that is the expected travel time that they will experience when traverse a freeway segment. From the point of view of ATMS the dynamic estimates of time dependencies in OD matrices is a major input to dynamic traffic models used for estimating the current traffic state and forecasting its short term evolution. Travel Time Forecasting and Dynamic OD Estimation are two of the key components of ATIS/ATMS and the quality of the results that they could provide depend not only on the quality of the models but also on the accuracy and reliability of the measurements of traffic variables supplied by the detection technology.
\end{abstract}

The quality and reliability of the measurements produced by traditional technologies, as inductive loop detectors, is not usually the required by real-time applications, therefore one wonders what could be expected from the new ICT technologies as for example Automatic Vehicle Location, License Plate Recognition, detection of mobile devices and so on. The main objectives of this paper are: to explore the quality of the data produced by the Bluetooth detection of mobile devices equipping vehicles for Travel Time Forecasting and its use to estimate time dependent OD matrices. Ad hoc procedures based on Kalman Filtering have been designed and implemented successfully and the numerical results of the computational experiments are presented and discussed.

Keywords: Travel Time, Origin Destination Matrices, Estimation Prediction, ATIS, ATMS

\section{INTRODUCTION}

Conceptually the basic architectures of Advanced Traffic Information Systems (ATIS) and Advanced Traffic Management Systems (ATMS) share the main model components; Figure 1 depicts schematically that of an integrated generic ATMS/ATIS:

- A road network equipped with a set of detection stations, suitably located on the network according to a detection layout that timely provide the data supporting the applications

- A Data Collection system collecting the raw real-time traffic data from sensors that must be filtered, checked and completed before being used by the models supporting the management system

- An ad hoc Historical Traffic Database storing the traffic data used by traffic models in combination with the real-time ones

- Traffic models aimed at estimating the current traffic state and short term forecasting it fed with real-time measured as well as processed data

- Advanced management models need time dependent Origin-Destination (OD) matrices, the algorithms for these applications combine the real-time and the historical data along with other non directly observable inputs (as the target OD matrices)

- Estimated and predicted states of the road network can be compared with the expected states if the comparison is OK (predicted and expected by the management strategies are close enough) then there is no action otherwise, depending on the differences found and on the envisaged control and management strategies to achieve the objectives a decision is made on which actions (traffic policies) will be the most appropriate to achieve the desired objectives. 
- Examples of such actions could be: ramp metering, speed control, rerouting, information on current status, levels of service, expected travel times and so on.

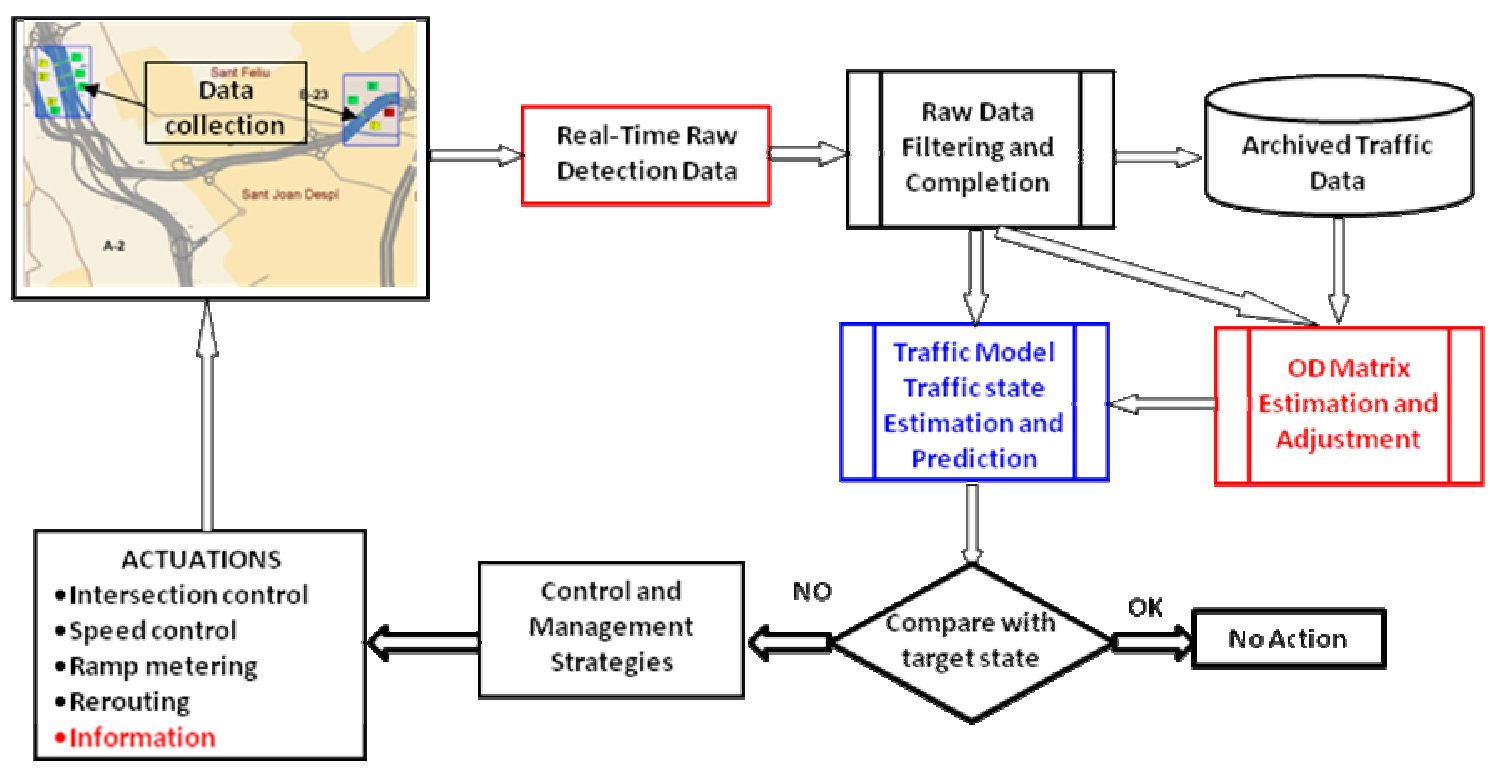

Figure 1: Conceptual approach to ATIS/ATMS architecture

The objective of this paper is the design an implementation of methods to support the forecasting of expected travel times and to estimate the time dependent OD matrices when, in addition to the usual data collection technologies, the network is equipped with sensors detecting vehicles equipped with Bluetooth mobile devices, i.e. hands free phones, Tom-Tom or Parrot devices and similar.

From the point of view of the information supplied by an ATIS to the motorists entering a freeway there is a wide consensus in considering as one of the most relevant the Forecasted Travel Time, that is the expected travel time that they will experience when traverse a freeway segment. From the point of view of ATMS the dynamic estimates of time dependencies in OD matrices is a major input to dynamic traffic models used for both estimate the current traffic state and forecast its short term evolution. Travel Time Forecasting and Dynamic OD Estimation are thus two of the key components of ATIS/ATMS and the quality of the results that they could provide depend on the quality of the models as well as on the accuracy and reliability of the traffic measurements of traffic variables supplied by the detection technology.

The quality and reliability of the measurements produced by traditional technologies, as inductive loop detectors, is not usually the required by real-time applications, therefore one wonders what could be expected from the new ICT technologies as for example Automatic Vehicle Location, License Plate Recognition, detection of mobile devices and so on. Consequently the main objectives of this paper are: to explore the quality of the data produced by the Bluetooth detection of mobile devices equipping vehicles for Travel Time Forecasting and its use to estimate time dependent OD matrices.

\section{CAPTURING TRAFFIC DATA WITH BLUETOOTH SENSORS}

The sensor integrates a mix of technologies that enable it to audit the Bluetooth and Wi-Fi spectra of devices within its coverage radius. It captures the public parts of the Bluetooth or Wi-Fi signals. Bluetooth is the global standard protocol (IEEE 802.15.1) for exchanging information wirelessly between mobile devices, using $2.4 \mathrm{GHz}$ short-range radio frequency bandwidth. The captured code consists in the combination of 6 alphanumeric pairs (Hexadecimal). The first 3 pairs are allocated to the manufacturer (Nokia, Panasonic, Sony...) and the type of manufacturer's device (i.e. phone, hands free, Tom-Tom, Parrot....) by the Institute of Electrical and Electronics Engineers (IEEE) and the last 3 define the MAC address, a unique 48-bit address assigned to each wireless device by the service 
provider company. The uniqueness of the MAC address makes possible to use a matching algorithm to $\log$ the device when becomes visible for the sensor. The logged device is time stamped and when it is logged again by another sensor at a different location the difference in time stamps can be used to estimate the travel time between both locations. Figure 2 illustrates graphically this process. A vehicle equipped with a Bluetooth device traveling along the freeway is logged and time stamped at time $t_{1}$ by the sensor at location 1. After traveling a certain distance is logged and time stamped again at time $t_{2}$ by the sensor at location 2. The difference in time stamps $\tau=t_{2}-t_{1}$ measures the travel time of the vehicle equipped with that mobile device, and obviously the speed assuming the distance between both locations is known. Data captured by each sensor is sent for processing to a central server by GPRS.

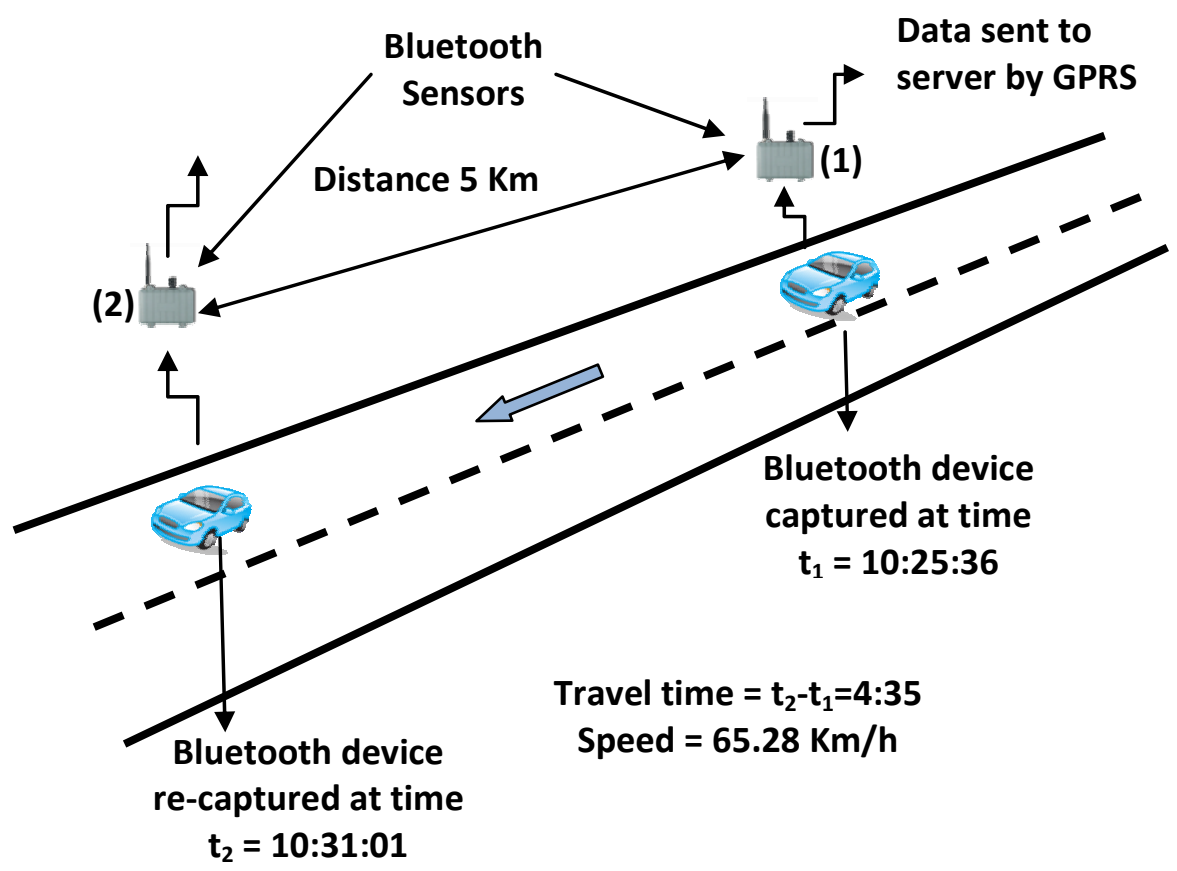

Figure 2: Vehicle monitoring with Bluetooth sensors

Raw measured data cannot be used without a pre-processing aimed at filtering out outliers that could bias the sample. A typical example could be case of a vehicle that stops at a gas station between the locations of the sensor. To remove these data from the sample a filtering process consisting of an adaptive mechanism has been defined, it assumes a lower bound threshold for the free flow speed $\mathrm{v}_{\mathrm{f}}$ in that section estimated by previous traffic studies, for example $70 \mathrm{Km} / \mathrm{h}$, which defines an upper bound $\tau_{\mathrm{f}}$ to the travel time between sensors at 1 and 2 in these conditions. Travel times larger than that threshold are removed as abnormal data. The system monitors every minute the aggregated average speed of the detected vehicles and if it is slowing down and getting closer to the threshold speed, for example average speed $-\mathrm{v}_{\mathrm{f}}<\alpha$, for example $\alpha=10 \mathrm{Km} / \mathrm{h}$, then the estimate of the speed threshold is decreased to $v_{f}-2 \alpha$, and the lower bound threshold for the section is updated accordingly. Smaller values of the average speeds (i.e. $60 \mathrm{Km} / \mathrm{h}$ ) could be interpreted in terms of a congestion building process and the threshold adaptation continues until a final value of $5 \mathrm{KM} / \mathrm{h}$. If the minute average speeds are increasing the process is reverted accordingly. In some especial conditions like an accident the changes in speed are not fluent and for these situations the rules are changed, if the system is unable to generate any match in more than 2 minutes, the range is open to a maximum time value $(5 \mathrm{Km} / \mathrm{h})$.

Since this sensor system can monitor the path of a vehicle, this could raise questions about the privacy of drivers. However, working with the MAC address of Bluetooth device ensures privacy, since the MAC address is not associated with any other personal data; the audited data cannot be related to 
particular individuals. Besides, so as to reinforce the security of data, an asymmetric encryption algorithm is applied before data leaves the sensor and gets to the database, making it impossible to recover the original data, (1).

\section{TRAVEL-TIME MEASUREMENTS AND FORECASTS}

A pilot project has been conducted in Barcelona (Spain) in a $40 \mathrm{Km}$. long section of the AP-7 Motorway, North of Barcelona, between Barcelona and the French border. The Figure 3 depicts graphically the site highlighting in different colors the various sections with limits represented by the colored circles correspond to the locations of the sensors used in the pilot. Sensors are located at mile posts at Km. 87.2, 91.3, 106.4, 119.2, 125.4 and 130.5 of the AP-7 Motorway.

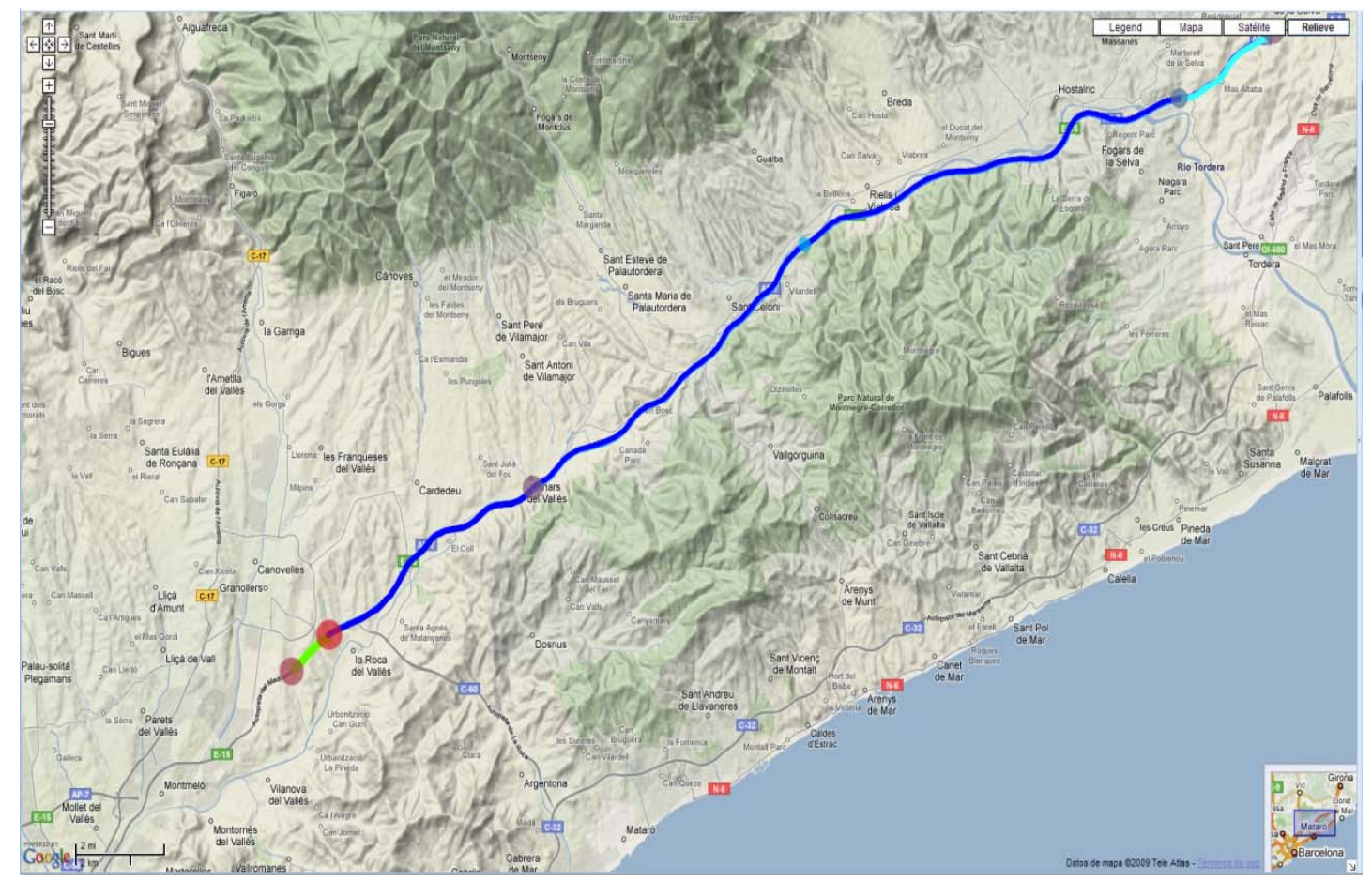

Figure 3: The site of the pilot project in the AP-7 Motorway in Barcelona

The Figure 4 depicts two examples of the measurements provided by the sensors located at the ends of the displayed motorway segment. The upper graphic, corresponding to direction south, towards Barcelona, displays in black the time evolution of the speeds between both locations along the day and in blue the number of detected devices. Table 2 presents an example of the raw data collected from the Bluetooth sensor, the column (id) identifies the temporal identity assigned by the encryption algorithm, time 1 and time 2 identify, respectively, the time stamps and the two last columns correspond to the calculated speeds and travel times.

\begin{tabular}{|c|c|c|c|c|}
\hline id & time1 & time2 & km & $t_{2}-t_{1}(\operatorname{seconds})$ \\
\hline 10483 & 11/06/2009 19:07 & $11 / 06 / 2009$ 19:24 & 149,24 & 989 \\
\hline 11925 & 11/06/2009 18:29 & 11/06/2009 18:47 & 133,33 & 1107 \\
\hline 12660 & 11/06/2009 18:48 & 11/06/2009 19:06 & 134,92 & 1094 \\
\hline 18419 & $11 / 06 / 2009$ 17:18 & 11/06/2009 17:40 & 113,89 & 1296 \\
\hline 18613 & 11/06/2009 19:35 & 11/06/2009 19:53 & 136,16 & 1084 \\
\hline & & & & \\
\hline
\end{tabular}

Table 1: Example of raw measured travel times $\left(\mathrm{t}_{2}-\mathrm{t}_{1}\right)$ and speeds 


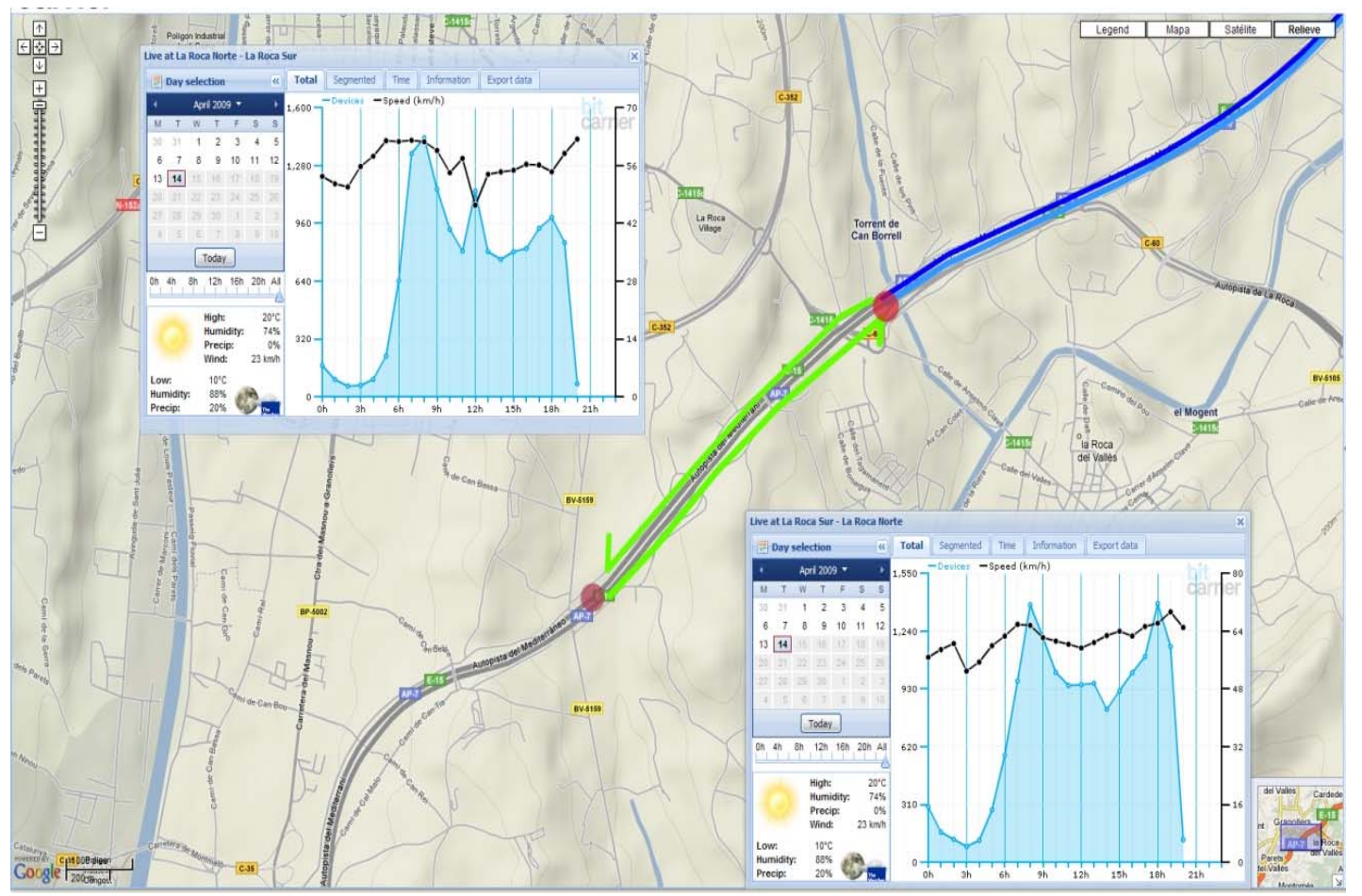

Figure 4: Two examples of Bluetooth detection: speeds and number of devices detected.

The data used for forecasting are the data filtered according to the adaptive procedure described above and aggregated by minute. Table 2 presents an example of the used data.

\begin{tabular}{|c|r|r|r|r|r|}
\hline \multicolumn{1}{c|}{ time } & total & phones & \multicolumn{1}{c|}{ cars } & Travel time (sec.) & Speed (Km/h) \\
\hline $2009-06-1117: 00$ & 4 & 0 & 4 & 1342 & 112,23 \\
\hline $2009-06-1117: 01$ & 8 & 4 & 4 & 1400 & 109,93 \\
\hline $2009-06-1117: 02$ & 2 & 1 & 1 & 1282 & 115,19 \\
\hline $2009-06-1117: 03$ & 7 & 5 & 2 & 1508 & 100,84 \\
\hline $2009-06-1117: 04$ & 4 & 3 & 1 & 1403 & 107,46 \\
\hline$\ldots \ldots \ldots \ldots \ldots \ldots \ldots$ & $\ldots \ldots \ldots$ & $\ldots \ldots \ldots$ & $\ldots \ldots \ldots$ & $\ldots \ldots \ldots \ldots \ldots \ldots \ldots$ \\
\hline
\end{tabular}

Table 2: Example of filtered data

Data have been collected for two months May and June 2009 which have been used to create a Historical Database. The modeling approach assumes that a Historic Database of past measurements and a real-time detection are available as shown in Figure 5.

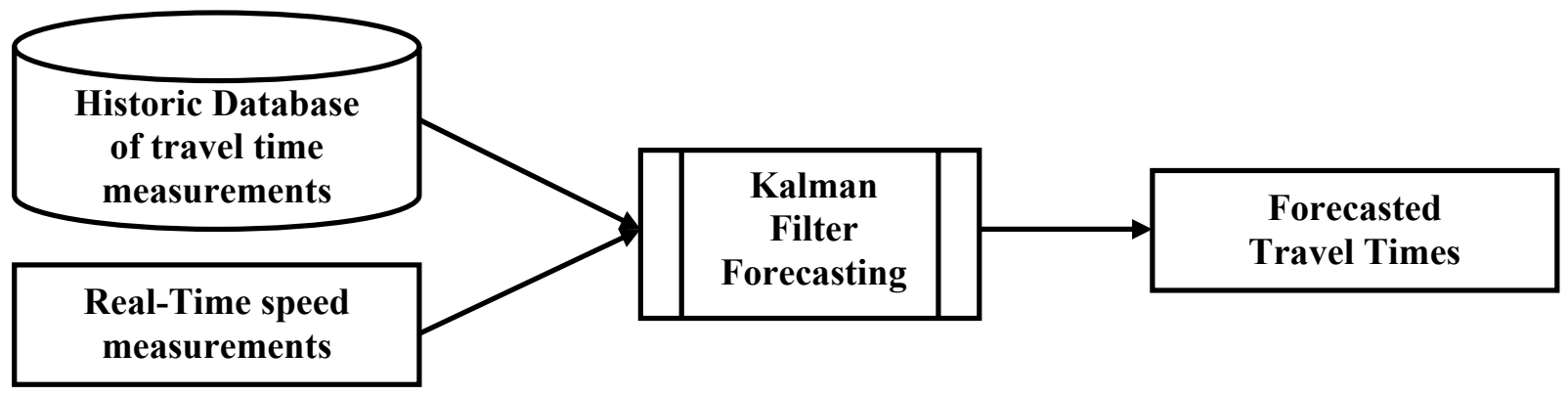

Figure 5: Conceptual scheme of the forecasting process 
Estimation and short term prediction of travel times is a key component of ATIS as has been highlighted in the introduction; consequently it has attracted the interest of researchers in recent years. A significant number of contributions deal with various methods, mostly based on applications of traffic flow theory, to achieve these objectives when measurements come from inductive loop detectors, the most widely used technology, but some other researchers have drawn their attention to cases when data are supplied by other technologies as probe vehicles (2), (3) or when cell phones or electronic toll identifications are the data sources, (4), (5), (6). In all these cases Kalman Filtering (7) has been proposed as the forecasting technique, it assumes that a system $\mathrm{S}$ is a state $\mathrm{E}_{\mathrm{k}}$ at time $\mathrm{k}$ defined by the values of the state variables $\mathrm{x}(\mathrm{k}) \in \mathfrak{R}$ at that time. The values of the state variables change in time according with the stochastic lineal equation in differences, transition equation:

$$
\mathrm{x}(\mathrm{k})=\mathrm{A}(\mathrm{k}) \mathrm{x}(\mathrm{k}-1)+\mathrm{w}_{\mathrm{k}}
$$

In our case $\mathrm{x}_{\mathrm{k}}$ is the travel time at time $\mathrm{k}, \mathrm{A}(\mathrm{k})$ is the Transition Function at time $\mathrm{k}$ and $\mathrm{w}_{\mathrm{k}}$ is a random error term representing the process error whose probability distribution is normal with mean zero and covariance $\mathrm{Q},[\mathrm{P}(\mathrm{w}) \sim \mathrm{N}(0, \mathrm{Q})]$. And a measurement $\mathrm{z}(\mathrm{k}) \in \mathfrak{R}$ related to the state variable $\mathrm{x}_{\mathrm{k}}$ by:

$$
\mathrm{z}(\mathrm{k})=\mathrm{H}(\mathrm{k})+\mathrm{v}_{\mathrm{k}}
$$

The measurement equation, where $\mathrm{H}(\mathrm{k})$ is the measurement fuction and $\mathrm{v}_{\mathrm{k}}$ is the measurement error whose probability distribution is also normal with mean zero and covariance $\mathrm{R},[\mathrm{P}(\mathrm{v}) \sim \mathrm{N}(0, \mathrm{R})]$. In our case $\mathrm{z}(\mathrm{k})$ will the travel time measured at time $\mathrm{k}$. To apply the Kalman Filtering to our case let's assume that $\hat{z}(\mathrm{k})$ is the data stored in the Historic Database, i.e. average values for time interval $\mathrm{k}$ for the traffic patterns corresponding to the traffic behavior for a particular day of the week and a time period, and $z(k)$ the real time measurement for time interval $t$, then the state estimation $\hat{x}(k)_{-}$for time period k made by the Kalman Filter is:

$$
\hat{\mathrm{x}}(\mathrm{k})_{-}=\mathrm{A}(\mathrm{k}-1) \hat{\mathrm{x}}(\mathrm{k}-1)_{+}
$$

Where $\hat{\mathrm{x}}(\mathrm{k})_{+}$is the Kalman prediction for time $\mathrm{k}$ given by:

$$
\hat{\mathrm{x}}(\mathrm{k})_{+}=\hat{\mathrm{x}}(\mathrm{k})_{-}+\mathrm{K}(\mathrm{k})\left[\mathrm{z}(\mathrm{k})-\hat{\mathrm{x}}(\mathrm{k})_{-}\right]
$$

The Kalman Gain $\mathrm{K}(\mathrm{k})$ is given by:

$$
\mathrm{K}(\mathrm{k})=\mathrm{P}(\mathrm{k})_{-}\left[\mathrm{P}(\mathrm{k})_{-}+\mathrm{R}(\mathrm{k})\right]^{-1}
$$

As a function of the estimate of the error covariance $\mathrm{P}(\mathrm{k})$. and $\mathrm{R}(\mathrm{k})$ the variance of vehicles' travel times in time interval $\mathrm{k}$. The error covariance $\mathrm{P}(\mathrm{k})$. is estimated in terms of the updating of the covariance error $\mathrm{P}(\mathrm{k}-1)_{+}$and the covariance of the process noise $\mathrm{Q}(\mathrm{k})$ as:

$$
\mathrm{P}(\mathrm{k})_{-}=\mathrm{A}(\mathrm{k}-1) \mathrm{P}(\mathrm{k}-1)_{+} \mathrm{A}(\mathrm{t}-1)+\mathrm{Q}(\mathrm{k})
$$

The update of the covariance error $\mathrm{P}(\mathrm{k})_{+}$is computed by:

$$
\mathrm{P}(\mathrm{k})_{+}=[1-\mathrm{K}(\mathrm{k})] \mathrm{P}(\mathrm{k})_{-}
$$

In what follows we will assume that $\mathrm{Q}(\mathrm{k})$ is zero and that $\mathrm{R}(\mathrm{t})$ can be estimated from the data in the database for each time interval. We will complete our model hypothesis assuming that the Transition Function $\mathrm{A}(\mathrm{k})$ is given as the ratios of historic travel times according with the identifies patterns stored in the Historic Database: 


$$
A(k)=\frac{\hat{z}(k)}{\hat{z}(k-1)}
$$

The resulting algorithm can be formalized in the following terms:

\section{Step 0. Initialization}

Set $\mathrm{k}:=0, \mathrm{~A}(0)=1$ and $\mathrm{P}(0)_{-}=\operatorname{Var}[\hat{z}(0)], \mathrm{N}=$ Number of Time Intervals

Step 1. Travel Time and Measurement Error Covariance Estimate

$$
\begin{aligned}
& \hat{\mathrm{x}}(\mathrm{k})_{-}=\mathrm{A}(\mathrm{k}-1) \mathrm{x}(\mathrm{k}-1)_{+} \\
& \mathrm{P}(\mathrm{k})_{-}=\mathrm{A}(\mathrm{k}-1) \mathrm{P}(\mathrm{k}-1)_{+} \mathrm{A}(\mathrm{t}-1)
\end{aligned}
$$

\section{Step 2. Kalman Gain Calculation}

$$
\mathrm{K}(\mathrm{k})=\mathrm{P}(\mathrm{k})_{-}\left[\mathrm{P}(\mathrm{k})_{-}+\mathrm{R}(\mathrm{k})\right]^{-1}
$$

\section{Step 3. Travel Time Prediction}

$$
\mathrm{K}(\mathrm{k})=\mathrm{P}(\mathrm{k})_{-}\left[\mathrm{P}(\mathrm{k})_{-}+\mathrm{R}(\mathrm{k})\right]^{-1}
$$

\section{Step 4. Measurement Error Covariance Update}

$$
\mathrm{P}(\mathrm{k})_{+}=[1-\mathrm{K}(\mathrm{k})] \mathrm{P}(\mathrm{k})_{-}
$$

Step 5. If $k=$ N Stop

Otherwise set $\mathrm{k}:=\mathrm{k}+1$ and repeat from 1 .

\section{ESTIMATION OF TIME DEPENDENT OD MATRICES}

\section{Data collection to estimate time dependent OD}

The possibility of tracking vehicles equipped with Bluetooth mobile raises naturally the question of whether this information can be used for estimating the dynamic or time dependent OD matrix whose entries $\mathrm{T}_{\mathrm{ij}}(k)$ represent the number of vehicles accessing the freeway at time interval $k$ by the entry ramp $i$ with destination the exit ramp $j$.

A simulation experiment has been conducted prior to deploy the technology for a pilot project. The selected site has been a $11.551 \mathrm{~km}$ section of the Ronda de Dalt, a urban freeway in Barcelona, between the Trinitat and the Diagonal Exchange Nodes. The site has 11 entry ramps and 12 exit ramps (including main section flows) in the studied section in direction Llobregat (to the south of the city), Figure 7 depicts a part of the site with the suggested sensoring layout. $D_{i}$ denotes the location of the $i$ th sensor at the main section; $E_{j}$ denotes the sensor located at the $j$-th entry ramp and $\mathrm{S}_{\mathrm{n}}$ the sensor located at the n-th exit ramp. Distance between detectors is shown in Figure 6.

The simulation emulates the logging and time stamping of a sample of equipped vehicles. Sensors are modeled located in each entry and exit ramps and in the main stream immediately after each ramp.

Bluetooth data are collected every second, and are matched when the same emulated MAC address is detected by different sensors along entry ramps, exit ramps and main section. As a result travel times between detectors can be obtained (Figure 2). Bluetooth and WiFi sensors can provide traffic counts and travel times between pair of sensors for any time interval up to 0.1 seconds for equipped vehicles. Since equipped vehicles data constitute a random sample of traffic data, travel times and OD pattern for equipped vehicles are real-time estimates of OD pattern and travel times for the whole population of vehicles.

Bluetooth and WiFi sensors can provide equipped counts every time interval at:

- On-ramps. 
- Off-ramps.

- Main section. In the simulated data for the Test-Site, main section sensors are located immediately after each ramp.

Bluetooth and WiFi sensors can provide travel times between any pair of sensors for equipped vehicles every time interval at:

- Travel times from each on-ramp sensor located entering the corridor to the off-ramp sensor exiting the corridor.

- Travel times from each on-ramp sensor located entering the corridor to any main-section sensor crossed for any equipped vehicle.

\begin{tabular}{|r|r|r|r|r|r|r|r|r|r|r|r|r|r|r|r|r|r|r|r|r|r|r|}
\hline From & E1 & D1 & D2 & D3 & D4 & D5 & D6 & D7 & D8 & D9 & D10 & D11 & D12 & D13 & D14 & D15 & D16 & D17 & D18 & D19 & D20 \\
\hline To & D1 & D2 & D3 & D4 & D5 & D6 & D7 & D8 & D9 & D10 & D11 & D12 & D13 & D14 & D15 & D16 & D17 & D18 & D19 & D20 & S12 \\
\hline Distance & 498 & 841 & 609 & 555 & 470 & 423 & 718 & 507 & 338 & 743 & 950 & 495 & 618 & 435 & 629 & 77 & 210 & 362 & 991 & 303 & 780 \\
\hline
\end{tabular}

Figure 6: Distance between main section detectors (in meters)
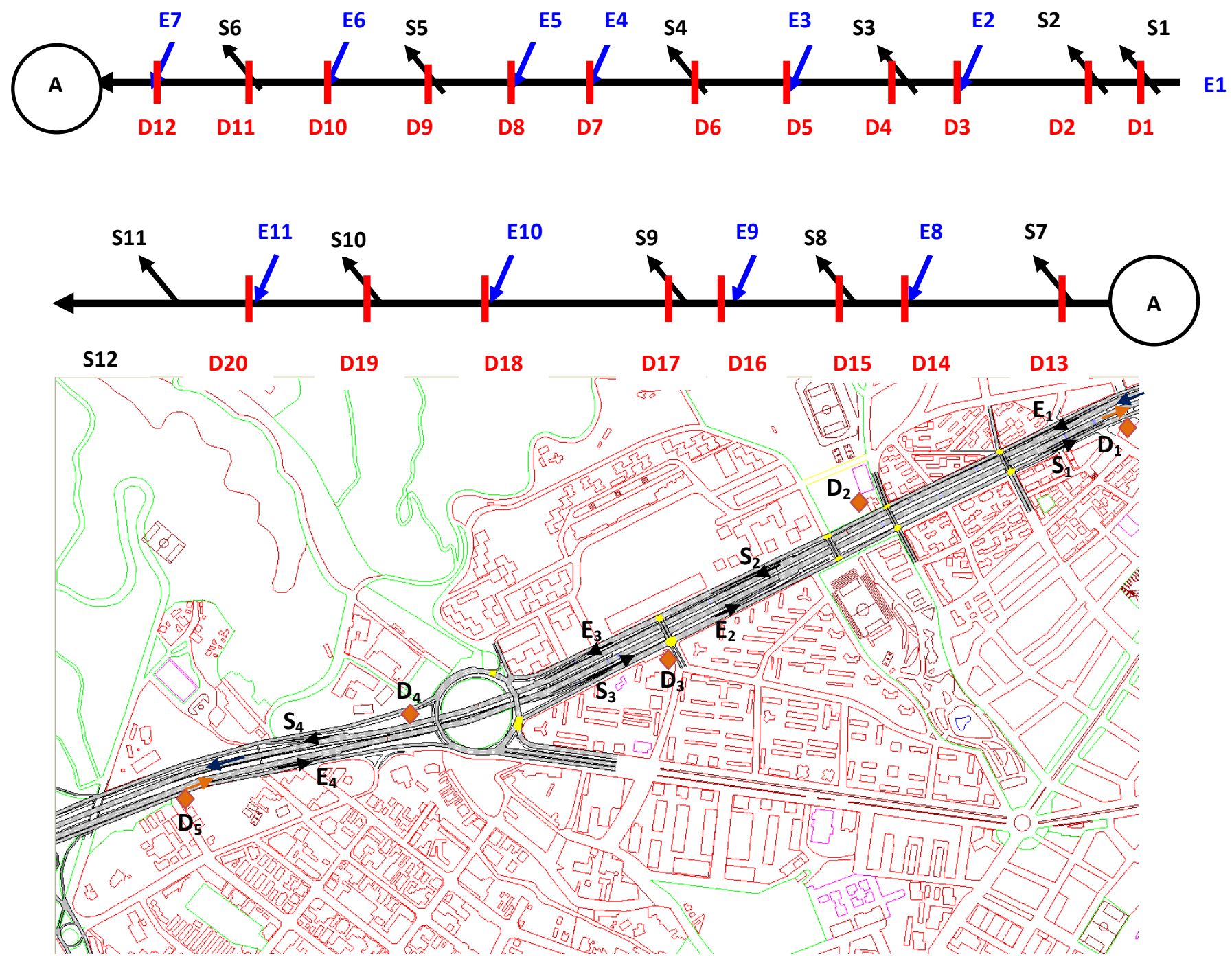

Figure 7: A segment of the site for the OD estimation showing part of the detection layout and diagram with the conceptual structure 
A natural question that could raise at this point would be: if Bluetooth sensors are tagging and time stamping vehicles entering the motorway by entry ramp $\mathrm{i}$ at time interval $\mathrm{k}$ and later on are tagged again when leave the motorway by exit ramp $\mathrm{j}$, then Bluetooth detection is generating a sample $\hat{\mathrm{T}}_{\mathrm{ij}}(\mathrm{k})$ of the number of vehicles entering the motorway by I during time interval $\mathrm{k}$ and later on leaving by $\mathrm{j}$, why don't use this sample to extrapolate to the whole population to estimate the time-dependent OD matrix $\mathrm{T}_{\mathrm{ij}}(\mathrm{k})$ ?. This is an interesting question that deserves further research but in the case of our computational experiments we compared the flow measurements of a couple of well calibrated inductive loop detectors located at the same points as the Bluetooth sensors with the number of vehicles detected by the Bluetooth sensors and we found that, although it was an apparently good matching between both counts of vehicles, as shown in Figure 8, a deeper analysis questioned the possibility of a straightforward expansion of the Bluetooth sample.
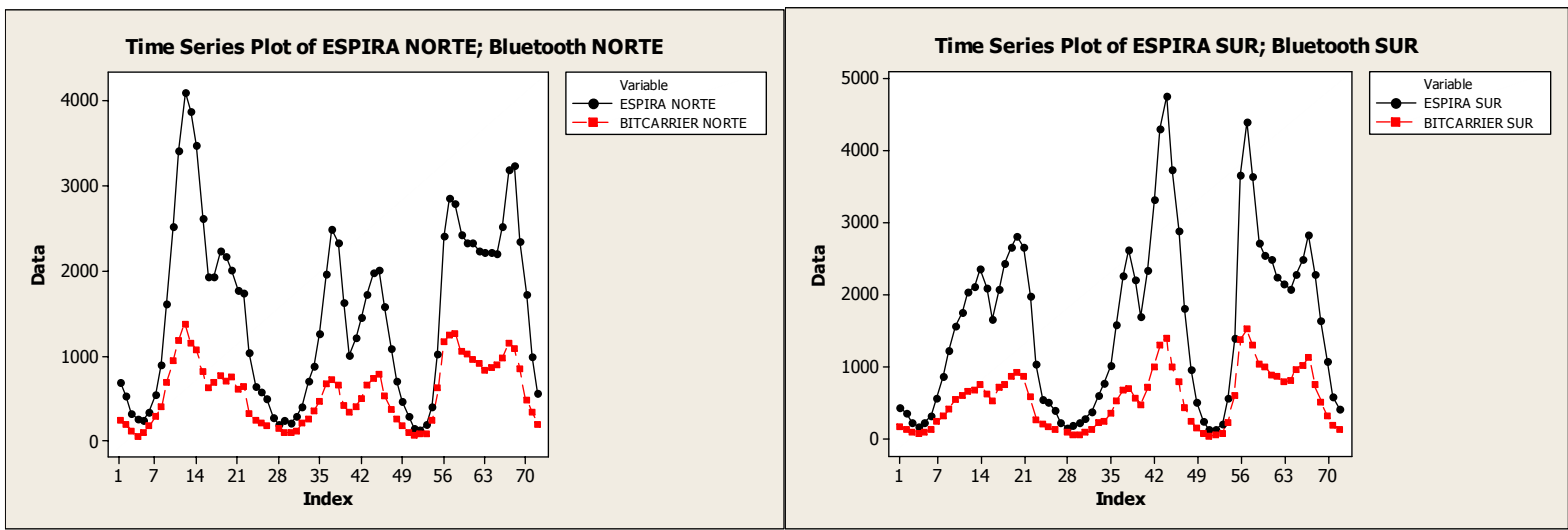

Figure 8: Comparison of Vehicles' Counts by Inductive Loop and Bluetooth

There are some facts that must be taken into account as for example that the sample sizes of Bluetooth equipped vehicles are not constant, change in time, depend on a penetration rate of the technology that is still growing and no stabilized. These facts lead to time varying errors that quite frequently are unacceptable as Figure 9 depicts.
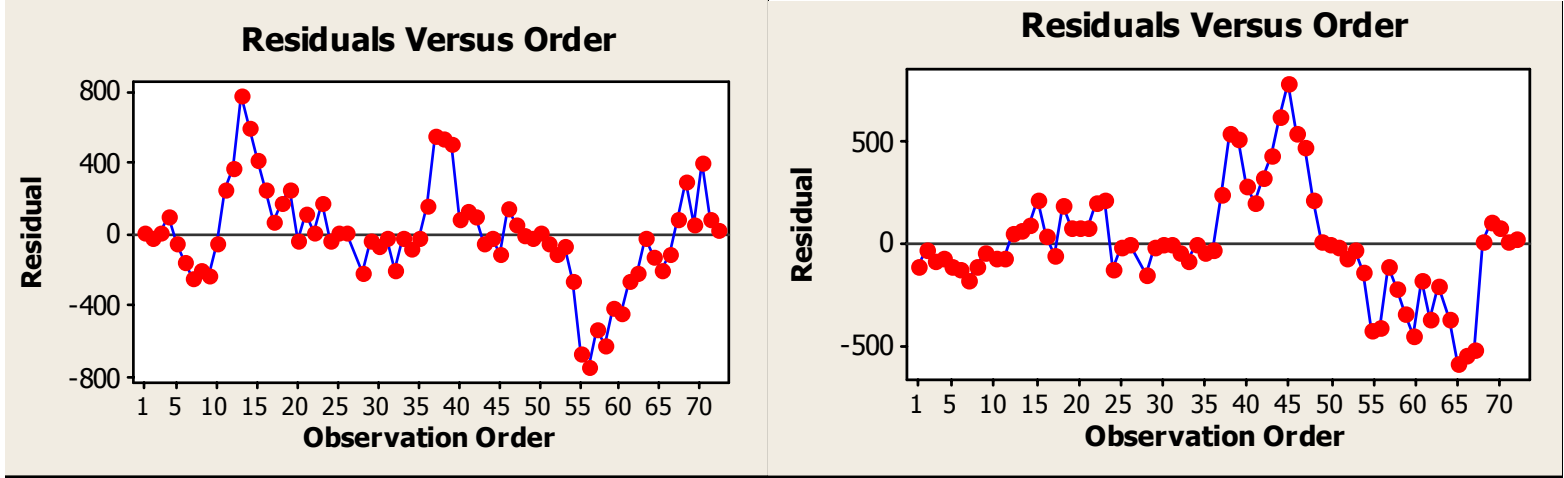

Figure 9: Residuals analysis for the comparison Inductive Loop and Bluetooth traffic counts

In consequence it is still risky to base a straightforward estimation of OD matrices based only on Bluetooth counting of vehicles but, on the other hand the accuracy in measuring speeds and travel times opens the door to the possibility of a more efficient use of Kalman Filtering for OD estimates, simplifying the equations and replacing state variables by measurements as described in the next section. 


\section{A Kalman Filter approach for estimating time dependent OD}

The estimation of OD matrices from traffic counts has received a lot of attention in the past decades. The extension to dynamic OD estimation in a dynamic system environment from time-series traffic counts has been proposed by many authors (8), (9) and (10). A review of the studies until 1991 is available in (11).

The system equations for OD estimation from static counts are underdetermined because there are far more OD pairs than number of equations, but since dynamic methods employ time-series traffic counts then more system equations than OD pairs are stated and a unique O-D matrix can thus be obtained. Both in the static and the dynamic methods the relations between OD matrices and traffic counts must be given. Static methods establish the relations between OD pairs and link flows through static traffic assignment models leading to entropy or bilevel mathematical programming formulation models, depending on the approach, that can be applied to linear corridors or urban networks, Spiess (12), Florian and Chen (13), Codina and Barcelo (14) . A crucial differentiation aspects between linear or network structures relies on the several paths available for networks (route choice is an essential aspect to be considered and proportions of OD flows using each of the OD available paths).

Most methods developed for dynamic OD estimation are limited to small networks or individual intersections due to the difficulty in establishing the relations between time-varying OD flows and link flows, even when no dynamic route choice has to be considered (on corridors), since dynamic traffic assignment procedures are not available, specially for congested situation. Effective methods for estimating OD matrices in urban network are subject of research, Ashok and Ben-Akiva(15), BenAkiva et al. (16), Mahmassani and Zhou (17).

We focus the attention on dynamic OD estimation in linear congested corridors where no route choice strategy is considered since there exists a unique path connecting each OD pair, but the travel time between each OD pair is considered and affected by congestion. If no congestion exits but a constant delay for each OD pair is considered the problem can be solved by any of the methods proposed by Bell (11) or Van Der Zijpp and Hamerslag (9); or Nihan and Davis (8) if OD travel times are negligible compared to counting interval.

Nihan and Davis (8) proposed a recursive method based on Kalman filter and state-space models where the state variables are the (constant or time dependent) OD proportions (between an entry and all possible destination ramps), the observation variables are exit flows on ramps for each interval and the relationship between the state variables and the observations includes a linear transformation where the numbers of departures from entry during time interval $k$ are explicitly considered. Sensors are assumed in all origins and destinations and provide time-varying traffic counts. Average RMS errors are presented for several algorithmic approaches. There are constraints in the OD proportions: non-negativity and each row of the matrix sum to 1 (the total number of vehicles entering the system will be equal to the total number of vehicles exiting the system). Unconstrained estimators are computed first and constraints are enforced later, several proposals are presented. The proposal is well-suited for intersections where OD travel-times are negligible compared to counting interval length.

Bell (11) formulates a space-state model and applies Kalman filter considering for each OD pair a fixed and non negligible OD travel time distribution where no counts on the main section are considered. Stability on traffic conditions is needed during the estimation process and arising congestion may not be captured in the formulation. A corridor with 4 entry ramps and 3 exit ramps is considered for 24 hours and a counting interval of 10 minutes.

Van Der Zijpp and Hamerslag (9) proposed an space-state model considering for each OD pair a fixed and non negligible OD travel time distribution where the state variables are time-varying OD proportions (between an entry and all possible destination ramps), the observation variables are main section counts for each interval, no exit ramp counts are present and the relationship between the state 
variables and the observations includes a linear transformation where the number of departures from each entry during time interval $k$ and a constant indicator matrix detailing OD pairs intercepted by each section detector are explicitly considered. Suggestions for dealing with structural constraints on state variables are proposed. The Kalman filter process is interpreted as a Bayesian estimator and initialization and noise properties are widely discussed. A $11 \mathrm{~km}$ corridor with 5 entry ramps, 5 exit ramps and 19 detectors is considered for 24 hours and a counting interval of 5 minutes. Tests with simulated data are undertaken for several methods and Kalman-based filter is indicated by authors to perform better than the other methods. Fixed OD travel time delays are not clearly integrated in the space state model, although is considered somehow by the authors.

Chang and $\mathrm{Wu}(10)$ proposed a space-state model considering for each OD pair a non fixed OD travel time estimated from time-varying traffic measures and traffic laws are implicitly included in the state variables. The state variables are thus time-varying OD proportions and fractions of OD trips that arrive each off-ramp $\mathrm{m}$ intervals after their entrance during interval $k$. The observation variables are main section and off-ramp counts for each interval and the relationship between the state variables and the observations is very complex and nonlinear. An Extended Kalman-filter approach is proposed and two algorithmic variants are implemented, one of them well-suited for on-line applications. A simple freeway section with 2 entries and 3 exits is used for numerical analysis. Parallel computing architectures are proposed to reduce computational time. Time interval length is recommended between 1 and 5 minutes.

We propose a space-state formulation for dynamic OD matrix estimation in corridors considering congestion that combines elements of Chang and Wu (10) and Van Der Zijpp and Hamerslag (9) proposals. A linear Kalman-based filter approach is implemented for recursive state variables estimation. Tracking of the vehicles is assumed by processing Bluetoooth and WiFi signals whose sensors are located in the entry ramps (mandatory), in the main section (as many as possible) and the off-ramps (as many as possible) as described in the above section. Traffic counts for every sensor and OD travel time from each entry ramp to the other sensors (main section and ramps) is available for any selected interval length higher than 1 second. Then travel time delays between OD pairs or between each entry and sensor location are directly provided by the detection layout and should no longer be state variables but measurements simplifying the approach and making it more reliable.

A basic hypothesis that requires a statistic contrast for test site applications is that equipped and non equipped vehicles are assumed to follow a common OD pattern. We assume that it holds in the following. Time interval length is suggested between 1 and 3 minutes to be able to detect arising congestion.

Consider a corridor section containing ramps and sensors numbered as in Figure 6. The notation is defined below:

$q_{i}(k) \quad$ : Number of equipped vehicles entering the freeway from on-ramp $i$ during interval $k$ and $i=1 \ldots I$

$s_{j}(k) \quad$ : Number of equipped vehicles leaving the freeway in off-ramp $j$ during interval $k$ and $j=1 \ldots J$

$y_{p}(k):$ Number of equipped vehicles crossing main section sensor $p$ and $p=1 \ldots P$

$G_{i j}(k):$ Number of vehicles entering the freeway from ramp $i$ during interval $k$ that are destined to off-ramp $j$

$g_{i j}(k)$ : Number of equipped vehicles entering the freeway from ramp $i$ during interval $k$ that are destined to off-ramp $j$

IJ : Number of feasible OD pairs depending on entry/exit ramp topology in the corridor, that is a maximum number of $I x J$

$t_{i j}(k)$ : Average measured travel time for equipped vehicles entering from entry $i$ and leaving offramp $j$ during interval $k$ 
$t_{i p}(k) \quad$ : Average measured travel time for equipped vehicles entering from entry $i$ and crossing sensor $\mathrm{p}$ during interval $k$

$b_{i j}(k) \quad:=g_{i j}(k) / q_{i}(k)$ the proportion of equipped vehicles entering the freeway from ramp $i$ during interval $k$ that are destined to off-ramp $j$.

$U_{i j q}^{h}(k):=1$ If the average measured time-varying travel time during interval $k$ to traverse the freeway section from entry $i$ to sensor q takes $\mathrm{h}$ time intervals, where $h=1 \ldots M$, $q=1 \ldots Q$ and $Q=J+P$ (the total number of main section and off-ramp sensors). $=0$ Otherwise

$e(k)=e:$ A fixed column vector of dimension I containing ones

$z(k) \quad$ : The observation variables during interval $k$; i.e. a column vector of dimension $\mathrm{I}+\mathrm{J}+\mathrm{P}$

The state variables are time-varying OD proportions for equipped vehicles entering the freeway from ramp $i$ during interval $k$ that are destined to off-ramp $j$. The observation variables are main section and off-ramp counts for each interval $k$. The relationship between the state variables and the observations involves a time-varying linear transformation that considers:

- The number of equipped vehicles entering from each entry during time interval $k, q_{i}(k)$.

- M time-varying indicator matrices, $\left[U_{i j q}^{h}(k)\right]$, detailing OD pairs intercepted by each sensor during interval $k$, entering the freeway $\mathrm{h}$ intervals before $k$; time-varying travel time measures are considered. Where $\mathrm{M}$ is the maximum number of intervals required for vehicles to traverse the entire freeway section considering a high congestion scenario.

The state variables $b_{i j}(k)$ are assumed to be stochastic in nature and evolve in some independent random walk process as shown in the state equation:

$$
b_{i j}(k+1)=b_{i j}(k)+w_{i j}(k)
$$

for all feasible OD pairs $(i, j)$ where $w_{i j}(k)$ 's are independent Gaussian white noise sequence with zero mean and covariance matrix $\mathbf{Q}$.

The structural constraints should be satisfied for the state variables,

$$
\begin{array}{cc}
b_{i j}(k) \geq 0 & i=1 \ldots I, \quad j=1 \ldots J \\
\sum_{j=1}^{J} b_{i j}(k)=1 & i=1 \ldots I
\end{array}
$$

Where $b(k)$ is the column vector containing all feasible OD pairs ordered by entry ramp; i.e, in the test site there are $I J=74$ OD pairs, less than the product $I x J=11 \times 12=132$. Equality constraint have been explicitly considered in the observation equations through the definition of dummy sums to 1 sensor counts where no measurement error is allowed as suggested but not tested in Van Der Zijpp and Hamerslag (9).

The column vector with the observation variables $z(k)$ has the following structure:

$$
z(k)^{T}=(s(k) \quad y(k) \quad e(k))^{T}
$$


The relationship between the state variables and the observations takes into account equality constraints for the current state variables $b_{i j}(k)$ as non-error dummy observations and a Gaussian error measurement for observed sensor counts during time interval $k$ :

$$
z(k)=\left(\begin{array}{c}
\mathbf{H}(\mathbf{k}) \\
\mathbf{E}
\end{array}\right) \mathbf{b}(\mathbf{k})+\left(\begin{array}{c}
v^{\prime}(k) \\
0
\end{array}\right)
$$

where $v_{i j}^{\prime}(k)$ 's are independent Gaussian white noise sequence with zero mean and covariance matrix $\mathbf{R}^{\prime}$, leading to a singular covariance matrix for the whole random noise vector $\mathbf{V}[v(k)]=\mathbf{R}=\left[\begin{array}{cc}\mathbf{R}^{\prime} & 0 \\ 0 & 0\end{array}\right]$. Dimension of $\mathbf{R}$ matrix is $(I+J+P)$.

Since the time varying travel times have to be taken into account to be able to model congestion, then time varying delays from entries to sensor positions have to be considered (they are described in the building process of the observation equations) and thus on ramp entry volumes for $\mathrm{M}+1$ intervals $k, k-1, \ldots, k-M$. State variables for intervals $k, k-1, \ldots, k-M$ are required to model interactions between time-varying OD patterns, counts on sensors and travel times delays from onramps to sensor positions.

Let $\mathbf{b}(\mathbf{k})$ be a column containing state variables for intervals $k, k-1, \ldots, k-M$ of dimension $(\mathrm{M}+1) \mathrm{xIJ}$.

$$
\mathbf{b}(\mathbf{k})^{T}=\left(\begin{array}{llll}
\mathrm{b}(\mathrm{k}) & \mathrm{b}(\mathrm{k}-1) & \ldots & \mathrm{b}(\mathrm{k}-M)
\end{array}\right)^{T}
$$

And the state equations have to be written using a matrix operator $\mathrm{D}$ for shifting one interval (following Chang and $\mathrm{Wu}(10)$ ), that allows eliminating the state variable for the last time interval (i.e., $k$-M) as:

$$
\mathbf{b}(\mathbf{k}+\mathbf{1})=\mathbf{D b}(\mathbf{k})+\mathbf{w}(\mathbf{k})
$$

where $\mathbf{w}(\mathbf{k})^{T}=\left(\begin{array}{llll}w(k) & 0 & \ldots & 0\end{array}\right)$ is a white noise sequence with zero mean and singular covariance matrix $\mathbf{V}[\mathbf{w}(\mathbf{k})]=\mathbf{W}=\left[\begin{array}{ll}\mathbf{Q} & \mathbf{0} \\ \mathbf{0} & \mathbf{0}\end{array}\right]$, where $\mathbf{Q}$ of dimension $I J$ has been previously defined (it is usually a diagonal matrix in most reported applications) and $\mathbf{D}=\left(\begin{array}{cccc}\mathbf{I}_{I J} & 0 & \ldots & 0 \\ \mathbf{I}_{I J} & 0 & 0 & 0 \\ 0 & \ddots & 0 & 0 \\ 0 & 0 & \mathbf{I}_{I J} & 0\end{array}\right)$

Let us detail in Eq. (15), the time-varying linear operator relating OD patterns and current observations for time interval $k$ in Eq. (4):

$$
\left(\begin{array}{c}
\mathbf{H}(\mathbf{k}) \\
\mathbf{E}
\end{array}\right)=\left(\begin{array}{cccc}
\mathbf{A} \mathbf{U}(\mathbf{k})^{\mathbf{T}} \mathbf{F}(\mathbf{k}) \\
\mathbf{B} & 0 & \ldots & 0
\end{array}\right)
$$


E : Matrix of row dimension I containing 0 for columns related to state variables in time intervals $k-1, \ldots, k-M$

B : Matrix of dimension IJ x I defining equality constraints (sum to 1 in OD proportions for each entry) for state variable in time interval $k$.

$\mathbf{F}(\mathbf{k})$ : Matrix of dimensions $(1+\mathrm{M}) \mathrm{IJ} \quad \mathrm{x} \quad(1+\mathrm{M}) \mathrm{IJ}$ consisting on diagonal matrices $f(k), \ldots, f(k-M)$ containing input on-ramp volumes that applies to each OD pair and time interval. Each $f($.$) is a squared diagonal matrix of dimension IJ.$

$\mathbf{g}(\mathbf{k}):$ Column vector of OD flows of equipped vehicles for time intervals $k, k-1, \ldots, k-M$

$\mathbf{U}(\boldsymbol{k})$ : Matrix of dimensions $(1+\mathrm{M}) \mathrm{IJ} \times(1+\mathrm{M})(\mathrm{J}+\mathrm{P})$ consisting on diagonal matrices $U(k), \ldots, U(k-M)$ containing an . For $U(k-h)$ is a matrix of dimensions $\operatorname{IJx}(\mathrm{J}+\mathrm{P})$ containing 1 if travel-time from entry $i$ to a given sensor $q$ takes $h$ intervals for vehicles captured by the $q$ sensor at time interval $k$ and 0 otherwise. Average mesured time varying travel times are critical and the clue for taking into account congestion effects.

A : Matrix of dimensions $(\mathrm{J}+\mathrm{P}) \times(1+\mathrm{M})(\mathrm{J}+\mathrm{P})$ that adds up for a given sensor $q$ (main section or off-ramp) traffic flows from any previous on-ramps arriving to sensor at interval $k$ assuming their travel time are $t_{i q}(k)$

$$
\mathbf{B}=\left(\begin{array}{cccc}
\overbrace{1 \ldots 1}^{I} & 0 & \ldots & 0 \\
0 & \overbrace{1 \ldots 1}^{I} & 0 & 0 \\
0 & 0 & \ddots & 0 \\
0 & 0 & 0 & \overbrace{1 \ldots 1}^{I}
\end{array}\right) \text { and thus } \mathbf{E} \mathbf{b}(\mathbf{k})=\left(\begin{array}{c}
\sum_{j} b_{1 j}(k) \\
\vdots \\
\sum_{j} b_{I j}(k)
\end{array}\right)=\left(\begin{array}{c}
1 \\
\vdots \\
1
\end{array}\right)=e
$$

$f(k)=\operatorname{diag}(\overbrace{q_{1}, \ldots q_{1}}^{J_{1}}, \overbrace{q_{2}, \ldots q_{2}}^{J_{2}}, \ldots, \overbrace{q_{I}, \ldots q_{I}}^{J_{I}})$ where $J_{i}$ is the number of possible off-ramps for entry $i$, for $i=1, \ldots, I$ and $\sum_{i=1, \ldots, I} J_{i}=I J$.

thus $\mathbf{F}(\mathbf{k}) \mathbf{b}(\mathbf{k})=\left(\begin{array}{ccc}f(k) & 0 & 0 \\ 0 & \ddots & 0 \\ 0 & 0 & f(k-M)\end{array}\right)\left(\begin{array}{c}b(k) \\ \vdots \\ b(k-M)\end{array}\right)=\left(\begin{array}{c}g(k) \\ \vdots \\ g(k-M)\end{array}\right)=\mathbf{g}(\mathbf{k})$

and $\mathbf{g}(\mathbf{k})$ is the column vector of OD flows of equipped vehicles for time intervals $k, k-1, \ldots, k-M$ and has dimension $(\mathrm{M}+1) \mathrm{IJ}$.

A matrix is composed by appending identity matrices of dimension $\mathrm{Q}=\mathrm{J}+\mathrm{P}$ for $\mathrm{M}+1$ times; i.e.

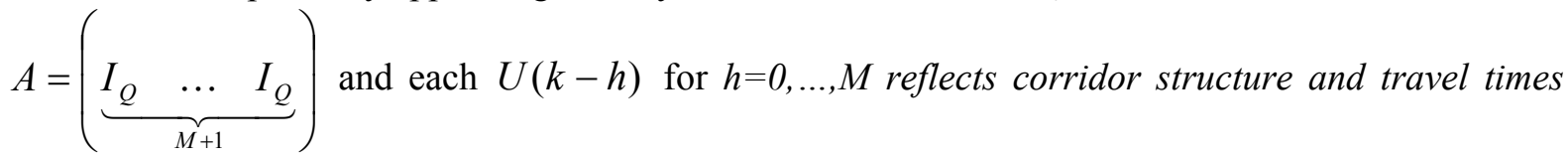
delays

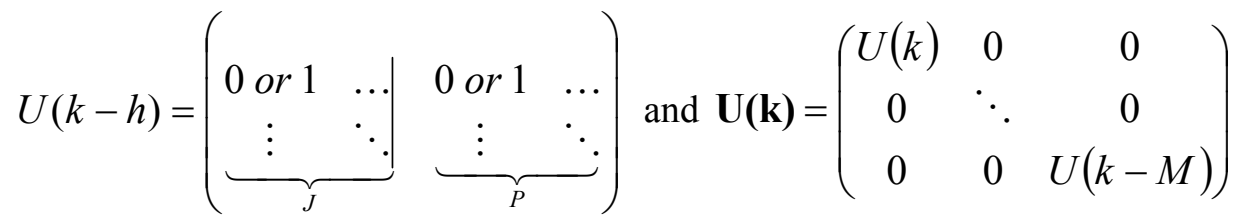


of dimensions $(1+\mathrm{M}) \mathrm{IJ} \times(1+\mathrm{M})(\mathrm{J}+\mathrm{P})$.

And thus, $\mathbf{H}(\mathbf{k}) \mathbf{b}(\mathbf{k})=\mathbf{A} \mathbf{U}(\mathbf{k})^{\mathbf{T}} \mathbf{F}(\mathbf{k}) \mathbf{b}(\mathbf{k}) \approx\left(\begin{array}{c}s(k) \\ y(k)\end{array}\right)$, the linear operator $\mathbf{H}(\mathbf{k})$ relates dynamic OD proportions, dynamic travel time delays and dynamic on-ramps entry flows with dynamic counts on sensors (main section and off-ramp) for equipped vehicles. The space-state formulation is almost completed,

$$
z(k)=\left(\begin{array}{c}
\mathbf{H}(\mathbf{k}) \\
\mathbf{E}
\end{array}\right) \mathbf{b}(\mathbf{k})+\left(\begin{array}{c}
v^{\prime}(k) \\
0
\end{array}\right)=\mathbf{R}(\mathbf{k}) \mathbf{b}(\mathbf{k})+v(k)
$$

A recursive linear Kalman-filter approach, well-suited for on-line applications, has been implemented in MatLab, using simulated data for the Test-Site. Matlab has been selected by its ability in performing algebraic operations with large matrices as the ones resulting from the approach taken.

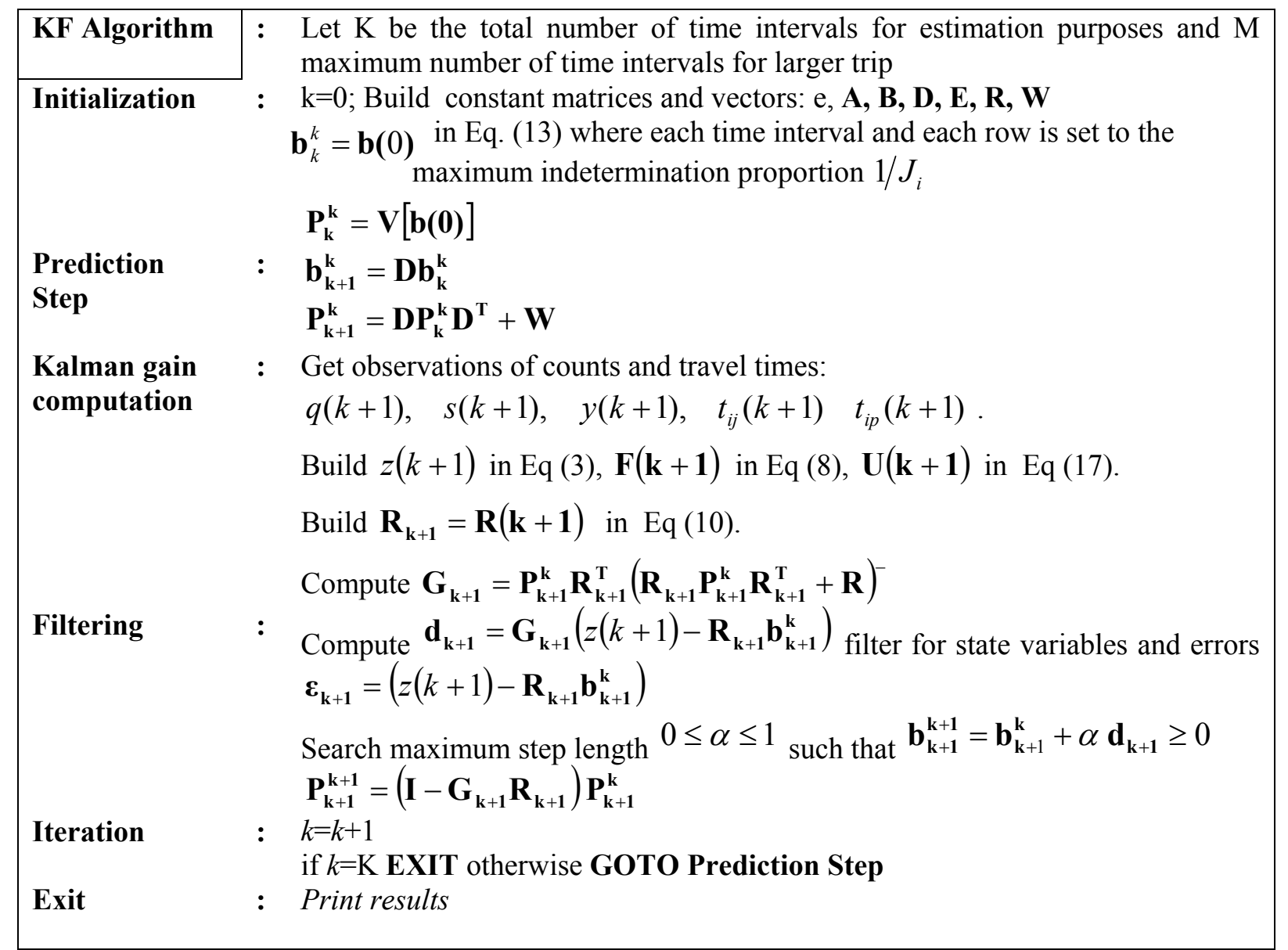

\section{PRELIMINARY RESULTS}

\section{Travel Time Forecasting}

Table 3 presents a sample of the results of applying the filter for five minutes Travel Time Forecasting in the conditions defined above. The computational results were obtained using the variances of the samples of 5 minutes for Tuesdays stored in the Historical Database and the real-time travel time measurements for a specific Tuesday. 
The fitting between the measured and predicted values is displayed in Figure 10. A quantitative estimation of the quality of the prediction is given by the correlation coefficient between the two series, $\mathrm{R}^{2}=0,9863$, and a Mean Absolute Relative Error of 0.0354. Taking into account that both series of data, measured and predicted travel times are time series an additional measure of how close they are can be defined in terms of Theil's coefficients (18).

\begin{tabular}{|c|c|c|c|c|c|c|c|c|}
\hline $\mathbf{k}$ & $\begin{array}{l}\text { Meas. Travel } \\
\text { Time }\end{array}$ & $\mathbf{R}(\mathbf{k})$ & $\mathbf{A}(\mathbf{k})$ & $\begin{array}{l}\text { State est. }= \\
A(k-1) * x(k-1)\end{array}$ & $\mathbf{P}(\mathbf{t})-$ & Kalman Gain & $\mathbf{P}(\mathbf{t})+$ & Predict. \\
\hline 0 & 325 & 7711 & 1 & 320 & 1000 & 0.1147 & 885.202617 & 320.5739 \\
\hline 1 & 306.4375 & 1600.7461 & 0.9428 & 320.5739 & 885.2026 & 0.3560 & 569.997534 & 315.5402 \\
\hline 2 & 359.5789 & 35845.2964 & 1.1734 & 297.5180 & 506.7457 & 0.0139 & 499.681694 & 298.3831 \\
\hline 3 & 314.0588 & 1199.9377 & 0.8734 & 350.1277 & 688.0151 & 0.3644 & 437.285992 & 336.9833 \\
\hline 4 & 332.4 & 2419.1733 & 1.0584 & 294.3237 & 333.5793 & 0.1211 & 293.156075 & 298.9378 \\
\hline 5 & 316.7778 & 1386.0617 & 0.9530 & 316.3959 & 328.3968 & 0.1915 & 265.493911 & 316.4690 \\
\hline & $\ldots$ & $\ldots \ldots$ & $\ldots$ & $\ldots$. & $\ldots$. & $\ldots$ & $\ldots$ & …....... \\
\hline
\end{tabular}

Table 3: Sequence of computations in the travel time forecasting algorithm

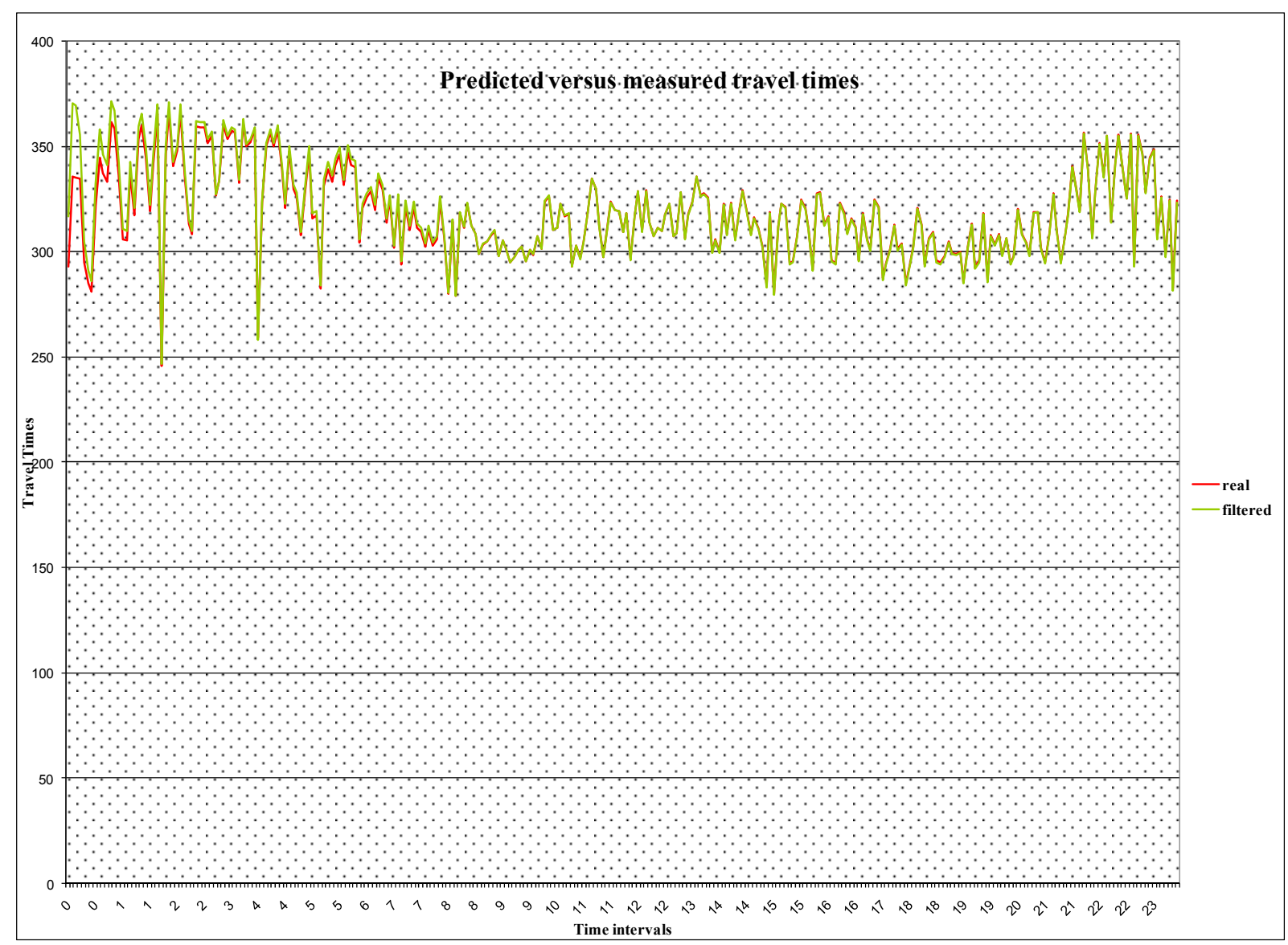

Figure 10: Predicted ("filtered") versus measured travel times

Theil's inequality coefficient is a measure on how close two time series are (overcoming the effect of outliers in RMS estimators) and is given by:

$$
\mathrm{U}=\frac{\sqrt{\frac{1}{\mathrm{n}} \sum_{\mathrm{i}=1}^{\mathrm{n}}\left(\hat{\mathrm{y}}_{\mathrm{i}}-\mathrm{y}_{\mathrm{i}}\right)^{2}}}{\sqrt{\frac{1}{\mathrm{n}} \sum_{\mathrm{i}=1}^{\mathrm{n}} \hat{\mathrm{y}}_{\mathrm{i}}^{2}+\sqrt{\frac{1}{\mathrm{n}} \sum_{\mathrm{i}=1}^{\mathrm{n}} \mathrm{y}_{\mathrm{i}}^{2}}}}
$$


Bounded between 0 and $1, \mathrm{U}=0$ can be interpreted as a perfect fitting between the two series, while $\mathrm{U}=1$ represents an unacceptable discrepancy. Values of $\mathrm{U}>0.2$ recommend to reject the predicted series. In Our case the value of $\mathrm{U}$ is $\mathrm{U}=0.02415735$ indicating that the matching is very good. On the other hand Theil's coefficient can be decomposed in the three coefficients:

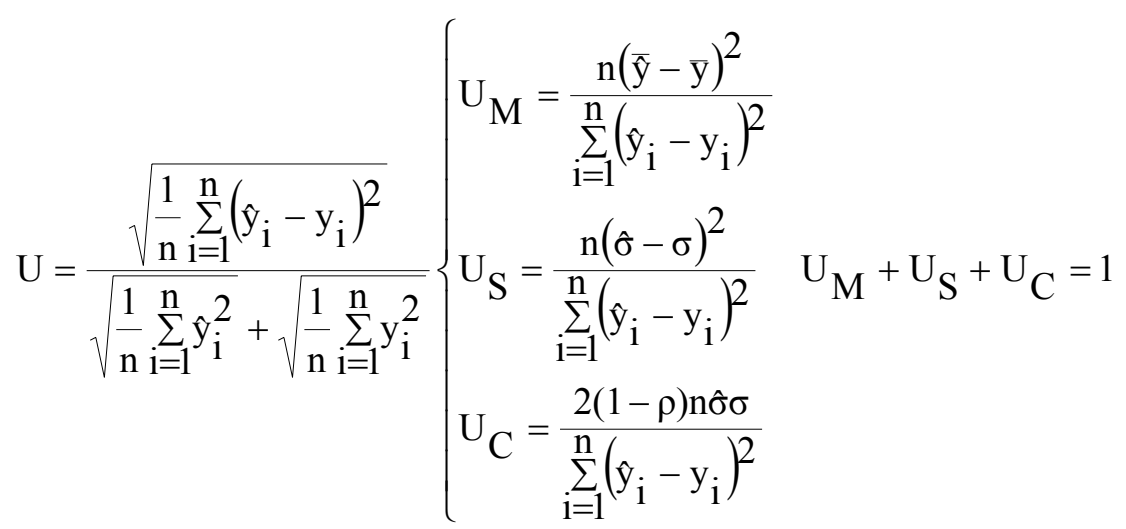

Where $\bar{y}$ and $y$ are, respectively, the means of the measurements and predictions, $\hat{\sigma}$ and $\sigma$ are the standard deviations and $\rho$ is the correlation coefficient. $\mathrm{U}_{\mathrm{M}}$, the bias proportion, can be considered as a measure of the systematic error, $U_{\mathrm{S}}$, the variance proportion, identifies the predicted series ability to reproduce the variability of the observed time series and $\mathrm{U}_{\mathrm{C}}$, the covariance proportion is a measure of the non systematic error. In our case the corresponding values are: $\mathrm{U}_{\mathrm{M}}=0,088641663$, $\mathrm{U}_{\mathrm{S}}=0,002415572$ and $\mathrm{U}_{\mathrm{C}}=0,913031427$. The small values of $\mathrm{U}_{\mathrm{M}}$ and $\mathrm{U}_{\mathrm{S}}$ certify the quality of the prediction.

\section{OD Estimation}

Two sets of computational experiments have been conducted. In the first a static OD pattern has been used for testing purposes, for a time horizon of 1 hour. OD pattern initialization is non-informative (every off-ramp of one on-ramp has the same probability). The test shows that the proposed Kalman Filtering approach converges successfully to the true results. Figure 11 depicts graphically the convergence progress for OD flows from entry 1 to each of the 12 off-ramps. The $\mathrm{x}$-axis correspond $\mathrm{s}$ to the iteration number and the y-axis the RMSE error value.

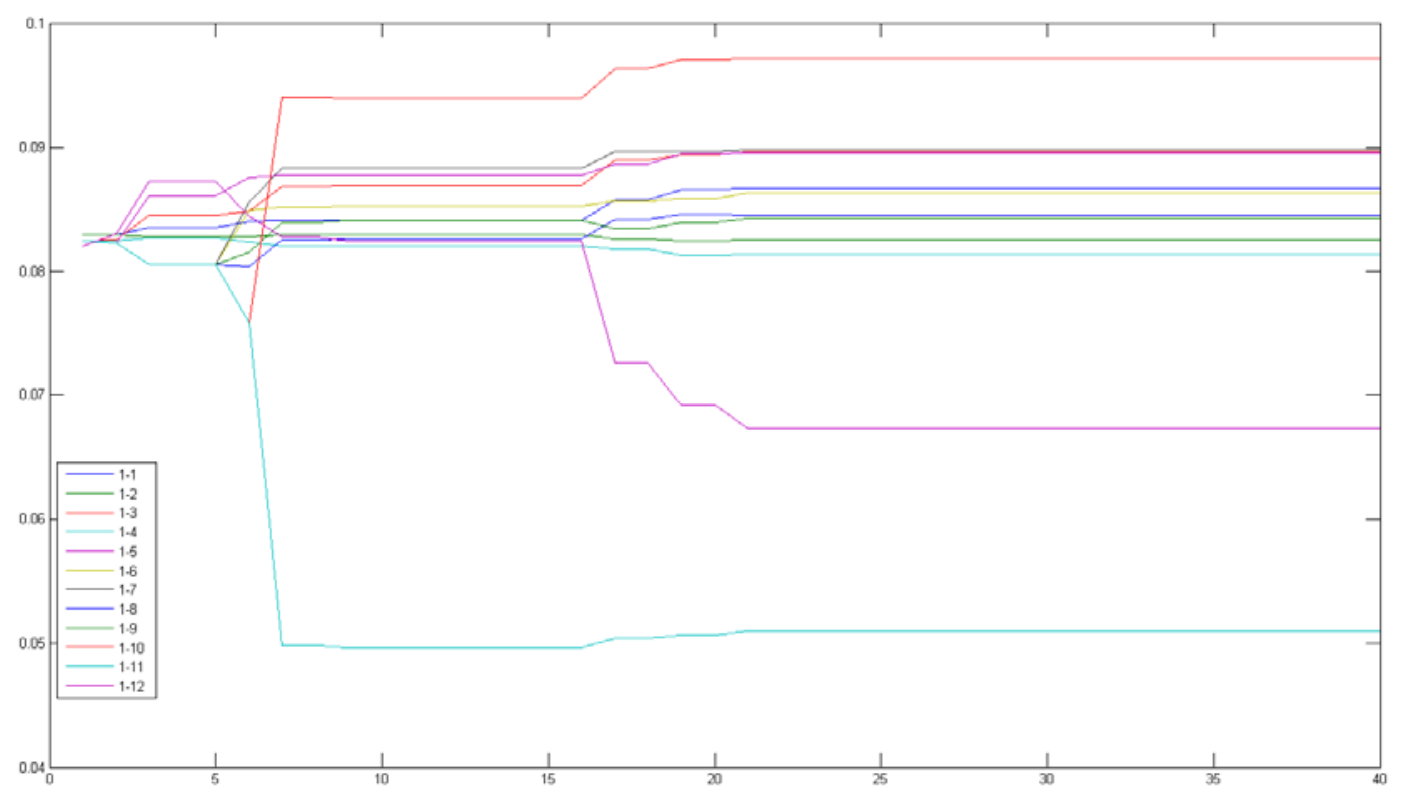


Figure 11. OD Pairs 1-1 to 1-12. Convergence to truly OD Proportion for constant OD Table 4 summarizes the values of the RMSE for each OD path flow at the end of the process.

\begin{tabular}{|c|c|c|c|c|c|c|c|c|c|c|c|c|}
\hline OD & $1-1$ & $1-2$ & $1-3$ & $1-4$ & $1-5$ & $1-6$ & $1-7$ & $1-8$ & $1-9$ & $1-10$ & $1-11$ & $1-12$ \\
\hline RMSEx $10^{3}$ & 0.96 & 0.55 & 1.2 & 0.17 & 0.91 & 0.73 & 0.2 & 0.14 & 0.32 & 0.65 & 0.53 & 1.5 \\
\hline
\end{tabular}

Table 4: Summary of RMSE values (multiplied by $10^{3}$ ) for a sample of OD pair flows

The Figure 12 illustrates a couple of additional cases for other paths flows from entries to exit ramps corresponding to shorter distances, (entry 3 to exit 5 and entry 4 to exit 7 respectively) the graphics show how in both cases the filter algorithm converges to the true values with RMSE values of 0.00143 for pair (3-5) and 0.0061 for pair (4-7).
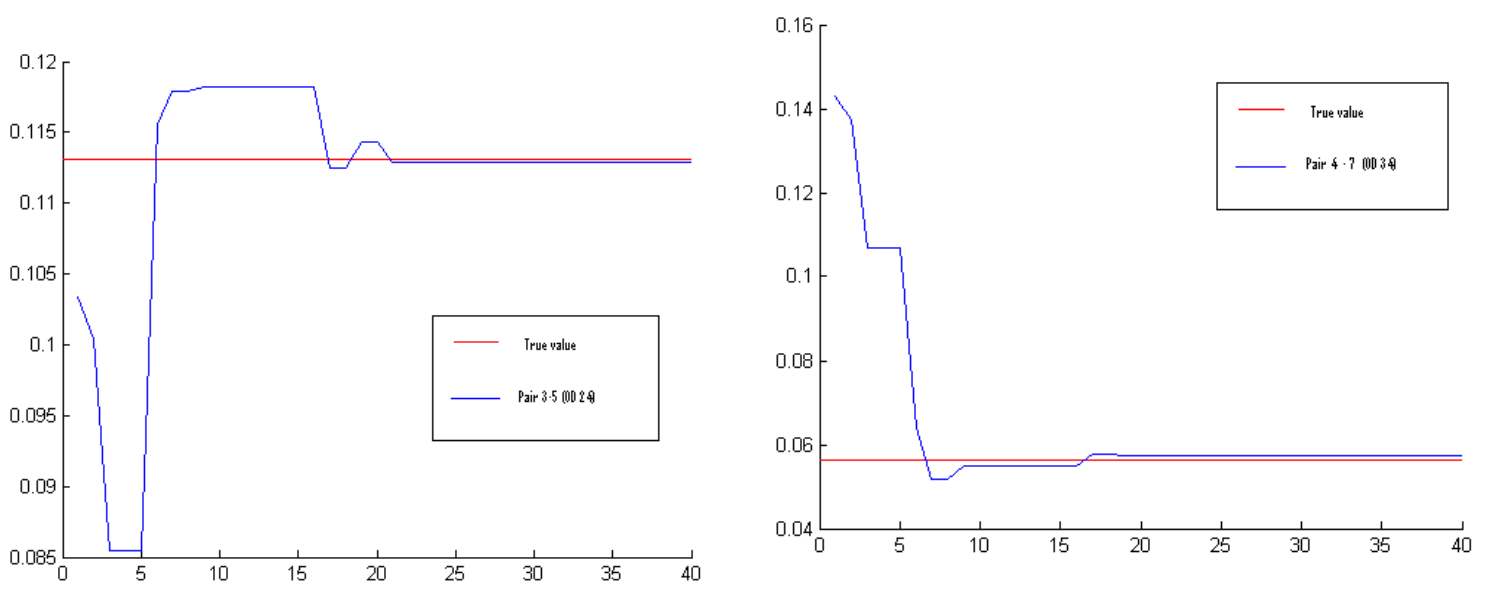

Figure 12: Convergence for OD pairs (3-5) left, and (4-7) right

The second set of computational experiments has been conducted with a time sliced OD matix totalizing the same demand as in the first set but the time horizon has been split in four time intervals of 15 minutes and the demand distributed to account for the $15 \%, 25 \%, 35 \%$ and $25 \%$ of the demand in each interval.

The results can be summarized as follows: for time intervals when traffic flow varies from free flow to dense but not yet conditions the filtering approach works acceptably well although its performance decreases as traffic flows become congested. The filtering approach does not work when traffic is heavily congested as a consequence of the amount of zero measurements. The results equivalent to those in Figure 11 for the same set of OD pairs are depicted in Figure 13. The x-axis, as before, correspond $\mathrm{s}$ to the iteration number and the y-axis the RMSE error value.

Table 5 summarizes the values of the RMSE for each OD path flow at the end of the process.

\begin{tabular}{|c|c|c|c|c|c|c|c|c|c|c|c|c|}
\hline OD & $1-1$ & $1-2$ & $1-3$ & $1-4$ & $1-5$ & $1-6$ & $1-7$ & $1-8$ & $1-9$ & $1-10$ & $1-11$ & $1-12$ \\
\hline RMSEx $10^{3}$ & 0.453 & 0.86 & 6.3 & 0.88 & 0.87 & 0.53 & 1.4 & 1.2 & 0.94 & 0.56 & 0.15 & 3.6 \\
\hline
\end{tabular}

Table 5: Summary of RMSE values (multiplied by $10^{3}$ ) for a sample of OD pair flows 


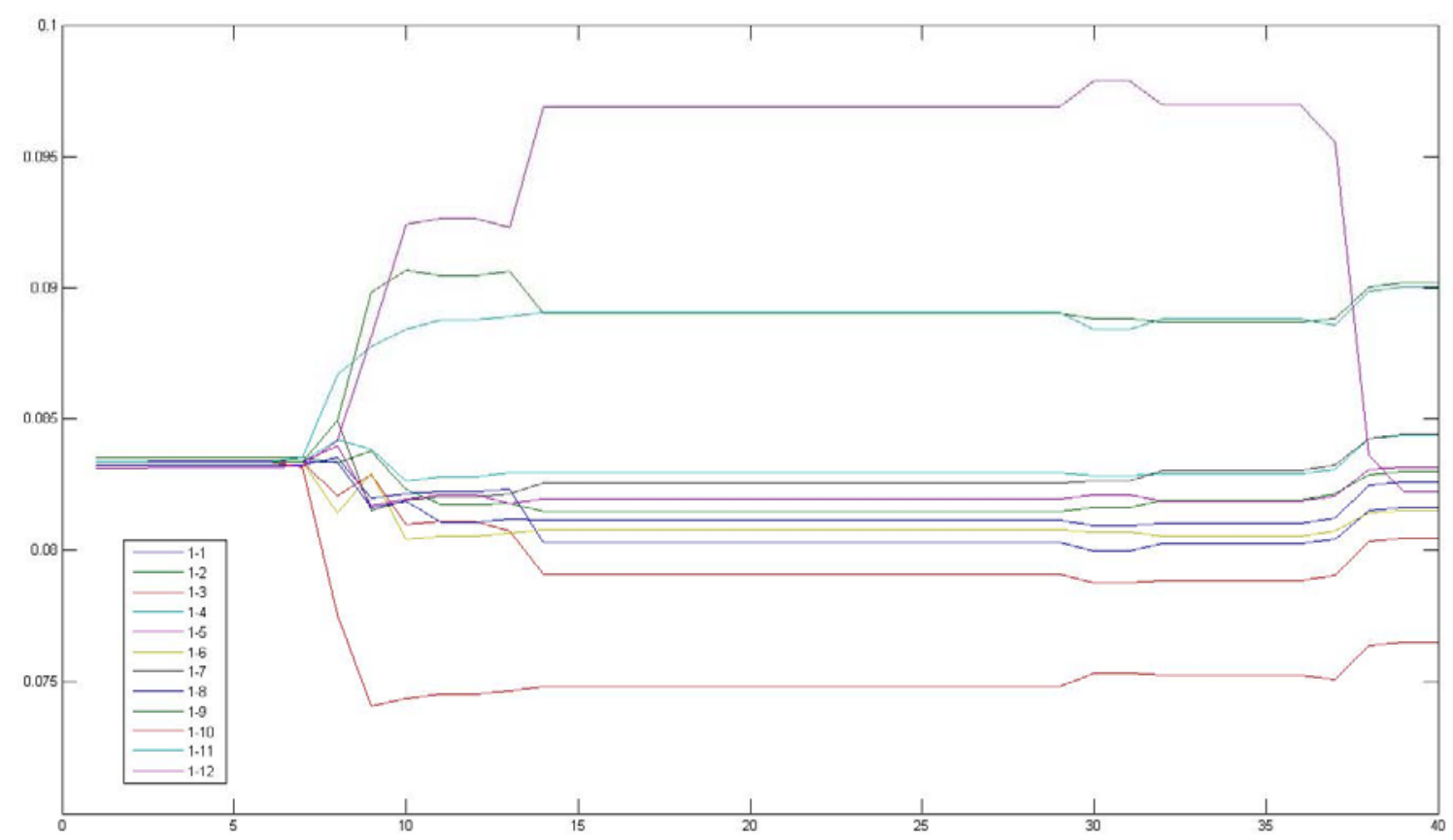

Figure 13: OD Pairs 1-1 to 1-12. Convergence to truly OD Proportions for time-sliced OD

\section{CONCLUSIONS AND FUTURE RESEARCH}

Bluetooth sensors to detect mobile devices have proved to be a mature technology that provides sound measurements of average speeds and travel times between sensor locations. These sensors are already in operation at the AP-7 Motorway in Spain between Barcelona and the French border. This paper has developed and tested a Kalman Filter approach for Travel Time Forecasting based on these measurements. The result proves the quality of the forecasts. We have also explored the combination of data available from this technology with other traffic data to develop another Kalman Filter approach to estimate dynamic origin to destination matrices in motorways. The results probe that the approach works fine in uncongested conditions and provides sound results. Further research is necessary to determine in which conditions the direct vehicle logging by Bluetooth can be used to estimate time-dependent OD matrices and how to deal with congested situations.

\section{ACKNOWLEDGEMENTS}

We acknowledge the firms Abertis, operator of the AP-7 Motorway, and Bitcarrier developer of the Bluetooth sensors for his kindness in making available the data used in this paper. And professor Pilar Muñoz, of the Department of Statistics and Operations Research at the Technical University of Catalonia for her kind advice on Kalman Filtering.

\section{REFERENCES}

(1)Sanfeliu, A., Punsola, A., Yoshimura, M. R Y., Llacer, M D. Gramunt, (2009), Legal challenges for networking robots deployment in european urban areas: the privacy issue, Workshop on Network Robots Systems; IEEE International Conference on Robotics and Automation (ICRA2009) Kobe

(2) Quiroga C. A. and D. Bullock, Travel time studies with global positioning and geographic information systems: an integrated methodology, Transportation Research Part C 6 (1998) 101127. 
(3) Nanthawichit, C., Nakatsuji, T., and Suzuki, H., Application of Probe Vehicle Data for Real-Time Traffic State Estimation and Short-Term Travel Time Prediction on a Freeway, Paper presented at the $82^{\text {nd }}$ TRB Meeting, Washington D.C.,2003.

(4) Kuchipudi C., Chien S.I.J., Development of a hybrid model for dynamic travel time prediction, Paper \# 03-2207, presented at the TRB,82 ${ }^{\text {nd }}$ Meeting, Washigton D.C., 2003

(5)Cheng, P., Zhijun Q., and Ran B., Particle Filter Based Traffic State Estimation Using Cell Phone Network Data, Proceedings of the Intelligent Transportation Systems Conference IEEE ITSC 2006.

(6) Chien S. I-Jy and Kuchipudi C. M., Dynamic Travel Time Prediction with Real-Time and Historic Data, Journal of Transportation Engineering, November/December 2003

(7) Kalman, R.E. (1960). A new approach to linear filtering and prediction problem. Journal of Basic Engineering 82 (1): 35-45.

(8) Nihan, N.L. and Davis, G.A., (1987). Recursive estimation of Origin. In: Destination matrices from Input/Output countsTransportation Research-B 21B, pp. 149-163 (2) .

(9) Van Der Zijpp, N.J. and Hamerslag, R., (1994). An improved Kalman filtering approach to estimate Origin-Destination matrices for freeway corridors. In: Transportation Research Records 1443, National Research Council, pp. 54-64.

(10) Chang, G.L. and Wu, J., (1994). Recursive estimation of time-varying Origin-Destination flows from traffic counts in freeway corridors. Transportation Research B Volume 28B No.2 pp. $141-160$

(11) Bell, M-G.H., (1991), The real time estimation of origin-destination flows in the presence of platoon dispersion, Transpn. Res. 25B, 115-125.

(12) Spiess H., (1990), A Gradient Approach for the O/D Matrix Adjustment Problem, Publication No. 693, Centre de Recherche sur les Transports, Université de Montréal.

(13) Florian M. and Chen Y., (1995), A Coordinate Descent Method for the Bi-level O/D Matrix Adjustment Problem, International Transactions in Operations Research, Vol. 2, No. 2, pp. 165175.

(14) Codina E., Barceló,J. (2004) Adjustment of O-D matrices from observed volumes: an algorithmic approach based on conjugate gradients, European Journal of Operations Research, Vol. 155 , pp. 535-557.

(15) Ashok, K. and Ben-Akiva, M., (2000) Alternative Approaches for Real-Time Estimation and Prediction of Time-Dependent Origin-Destination Flows, Transportation Science 34(1), 21-36.

(16) Ben-Akiva, M., Bierlaire, M., Burton, D., Kotsopoulos, H.N. Mishalani, R., (2001), Network State Estimation and Prediction for Real-Time Traffic Management, Networks and Spatial Economics, 1, pp. 293-318.

(17) Mahmassani, H.S., and Zhou, X., (2005), Transportation System Intelligence: Performance Measurement and Real-Time Traffic Estimation and Prediction in a Day-to-Day Learning Framework, Chapter 16 in Advances in Control, Communication Networks, and Transportation Systems, In Honor of Pravin Varaiya, edited by E. Abed, Birkhauser,

(18) H. Theil, Applied Economic Forecasting, North-Holland, 1966 